\title{
Age-Related Changes in the Photoreactivity of Retinal Lipofuscin Granules: Role of Chloroform-Insoluble Components
}

\author{
Matgorzata Różanowska, ${ }^{1,2}$ Anna Pawlak, ${ }^{1}$ Bartosz Różanowski, ${ }^{1,2,3}$ Christine Skumatz, ${ }^{4}$ \\ Mariusz Zareba, ${ }^{1}$ Mike E. Boulton, ${ }^{2}$ Janice M. Burke, ${ }^{4}$ Tadeusz Sarna, ${ }^{1}$ and \\ John D. Simon ${ }^{5}$
}

PurPose. Lipofuscin accumulates in human retinal pigment epithelium (RPE) cells with age and may be the main factor responsible for the increasing susceptibility of RPE to photooxidation with age. As the composition, absorption, and fluorescence of lipofuscin undergo age-related changes, the purpose of this study was to determine whether photoreactivity of lipofuscin granules also changes with the donor age.

Methods. To determine whether the photoreactivity of lipofuscin itself is age related, lipofuscin granules were isolated from human RPE and pooled into age groups. Photoreactivity was assessed by measuring action spectra of photo-induced oxygen uptake and photogeneration of reactive oxygen species. Separation of chloroform-soluble (ChS) and -insoluble (ChNS) components by Folch's extraction was used to determine the factors responsible for the age-related increase in lipofuscin photoreactivity.

Results. The observed rates of photo-induced oxygen uptake and photo-induced accumulation of superoxide-derived spin adducts indicated that when normalized to equal numbers of lipofuscin granules, aerobic photoreactivity of lipofuscin increased with age. Both ChS and ChNS mediated photogeneration of singlet oxygen, superoxide radical anion, and photooxidation of added lipids and proteins. Although both ChS and ChNS exhibited substantial photoreactivities, neither exhibited significant age-related changes when normalized to equal dry

From the ${ }^{1}$ Department of Biophysics, Faculty of Biotechnology, Kraków, Poland; the ${ }^{2}$ School of Optometry and Vision Sciences, Cardiff University, Cardiff, United Kingdom; the ${ }^{3}$ Department of Cytology and Genetics, Institute of Biology, Pedagogical Academy, Kraków, Poland; the ${ }^{4}$ Department of Ophthalmology, Medical College of Wisconsin, Milwaukee, Wisconsin; and the ${ }^{5}$ Departments of Chemistry and Ophthalmology, Duke University, Durham, North Carolina.

Presented at the annual meeting of the Association for Research in Vision and Ophthalmology, Fort Lauderdale, Florida, May 2002.

Supported by State Committee for Scientific Research, Poland (KBN) Grants 3P04A 04423, 3P04A 08022 and 4P05A 03615; Grants R01 EY13722, R01 EY10832, and P30 EY01931 from the National Eye Institute; by an unrestricted grant from Research to Prevent Blindness to the Medical College of Wisconsin; and the Wellcome Trust, United Kingdom and by IRIS Fund, London, United Kingdom. BR and MR are recipients of Wellcome Trust Traveling Wellcome Fellowships. MR is a recipient of the Polish-United States Fulbright Commission Senior Fulbright Fellowship.

Submitted for publication March 18, 2003; revised August 30, 2003; accepted November 7, 2003.

Disclosure: M. Różanowska, None; A. Pawlak, None; B. Różanowski, None; C. Skumatz, None; M. Zareba, None; M.E. Boulton, None; J.M. Burke, None; T. Sarna, None; J.D. Simon, None

The publication costs of this article were defrayed in part by page charge payment. This article must therefore be marked "advertise$m e n t "$ in accordance with 18 U.S.C. $\$ 1734$ solely to indicate this fact.

Corresponding author: Małgorzata Różanowska, School of Optometry and Vision Sciences, Cardiff University, Redwood Building, King Edward VII Avenue, Cardiff CF10 3NB, UK; rozanowskamb@cf.ac.uk. mass. In contrast, ChNS contents in lipofuscin granules significantly increased with aging.

Conclusions. Aerobic photoreactivity of RPE lipofuscin substantially increases with aging. This effect may be ascribed to the increased content of insoluble components. (Invest Ophthalmol Vis Sci. 2004;45:1052-1060) DOI:10.1167/iovs.030277

Tn developed Western countries, the leading cause of pro1 gressive visual loss in the elderly is age-related macular degeneration. One of the risk factors for the development of this disease is chronic exposure to blue light, presumably mediated by endogenous chromophores. ${ }^{1}$ A main suspect is lipofuscin, a lipid and protein aggregate containing several fluorophores and photosensitizer(s) that accumulates with age in human retinal pigment epithelium (RPE). ${ }^{1,2}$ On photoexcitation, lipofuscin generates reactive oxygen species, ${ }^{3-8}$ is phototoxic to RPE cells in culture, ${ }^{9}$ and is one of the major contributing factors to the age-related increase in aerobic photoreactivity of RPE cells. ${ }^{4}$

Lipofuscin photoreactivity has been studied mainly in whole lipofuscin granules or chloroform-soluble (ChS) components obtained by Folch's extraction. Both lipofuscin granules and ChS components mediate photo-induced oxygen uptake, whose efficiency increases steeply with decreasing irradiation wavelength. ${ }^{40}$ In lipofuscin granules, the oxygen uptake is accompanied by oxidation of intra- and extragranular lipids and proteins. ${ }^{11}$ This oxidation may be mediated by any of a number of active intermediates. Photoexcited granules generate singlet oxygen, superoxide radical anion, hydrogen peroxide, and lipid hydroperoxides. ${ }^{4}$ Lipofuscin's ChS components contain photosensitizer(s $)^{5-7}$ that, on excitation with UV or blue light, form a triplet state with high enough energy to form singlet oxygen. ${ }^{5,7}$ They also photosensitize formation of free radicals, including superoxide. 6

During Folch's extraction a considerable fraction of lipofuscin remains insoluble as a brownish layer between the chloroform- and methanol-PBS-enriched phases. Although photoinduced oxygen uptake in a suspension of this fraction exhibits increasing efficiency with decreasing irradiation wavelength, ${ }^{10}$ its role in photoreactivity of lipofuscin granules is unclear.

Despite considerable research effort, the chemical identity of the main photosensitizer(s) present in lipofuscin remains unknown. One of the components of lipofuscin that has been considered as a potential photosensitizer is the orange fluorescent pyridinium bisretinoid, A2E. ${ }^{9,12}$ However, even though A2E has been shown to be phototoxic to RPE cells in vitro, ${ }^{13-16}$ its role in lipofuscin phototoxicity and photoreactivity is only minor. ${ }^{9,10,17-19}$ It is also not known whether the primary chromophores are in the ChS or insoluble fraction, or both.

Not only does the number of granules in the RPE increase with age, but also the granule composition changes with age, with the protein-to-lipid ratio increasing from approximately 
1.5 to 3.0 for lipofuscin isolated from donors younger than 40 and older than 47 years of age, respectively. ${ }^{20}$ Age-related changes also occur in the absorption and fluorescence properties of lipofuscin granules, ${ }^{21,22}$ suggesting that other photochemical properties, and therefore photoreactivity, may undergo age-related changes as well. The purpose of the present study was to compare the photoreactivity of lipofuscin granules from different age groups and to determine whether ChS or chloroform-insoluble (ChNS) components of lipofuscin are responsible for any age-related changes.

\section{Materials ANd Methods}

\section{Chemicals}

Chemicals, of reagent grade or better, were purchased from SigmaAldrich Chemie GmbH (Steinheim, Germany), Sigma-Aldrich (St. Louis, MO), or Merck (Darmstadt, Germany) and used as supplied. 4-Protio3-carbamoyl-2,2,5,5-tetraperdeuteromethyl-3-pyrroline-1-yloxy $\quad(\mathrm{mH}-$ CTPO) was a gift from Howard J. Halpern (University of Chicago, Chicago, IL). Phosphate-buffered saline (PBS) without calcium and magnesium was treated with chelating resin (Chelex 100; Sigma-Aldrich) before use, to minimize the content of metal ions.

\section{Isolation of Lipofuscin, Melanolipofuscin, and Melanosome Granules}

Human eyes were obtained from the Wisconsin Lions Eye Bank. RPE cells were isolated as described previously ${ }^{4}$ and kept frozen at $-70^{\circ} \mathrm{C}$ until sufficient material was collected for isolation of pigment granules. To study age-related changes in lipofuscin photoreactivity, RPE cells were pooled according to donor age into four age groups: younger than 40 (32 pairs of eyes; age range, 1- 40 years), 41 to 60 (37 pairs), 61 to 80 ( 47 pairs), and older than 81 ( 44 pairs; age range, $81-98$ years of age). For some comparative experiments between ChS and ChNS components of lipofuscin, lipofuscin granules were isolated from RPE cells pooled from donors aged more than 40 years. Pigment granules were isolated and purified from RPE cells collected from pooled samples, as previously described. ${ }^{4}$ Isolated granules were suspended in PBS ( $\mathrm{pH} 7.2$ ) and dispersed by forcing them through a narrow-gauge needle. Concentration of the human granules was determined by counting in a hemocytometer. Typical concentration of the pigment granules used in experiments was in the range of 0.5 to $4.0 \times 10^{9} / \mathrm{mL}$. Aliquots of the lipofuscin suspension were set aside for experiments on whole lipofuscin granules. The remaining lipofuscin suspensions were used for Folch's extraction procedure. In some experiments, lipofuscin granules from the 41- to 60- and 61- to 80-year age groups were combined.

\section{Extraction of Lipofuscin}

Lipofuscin granules were extracted according to a modified Folch's procedure. ${ }^{23}$ Suspensions of lipofuscin granules in PBS were vortexed for 15 seconds in the presence of chloroform-methanol (2:1, vol/vol) mixture (5:8, PBS:chloroform-methanol mixture, vol/vol), followed by centrifugation. The upper aqueous phase and lower chloroform phase were withdrawn separately and further purified by repeated extraction in triplicate. The interphase material was subjected to similar treatments in triplicate with PBS-chloroform-methanol. After the final centrifugation, appropriate phase or interphase material was collected in preweighed glass flasks, dried in a vacuum and weighed to determine the dry mass. An aliquot of a selected solvent-PBS, chloroform, dimethyl sulfoxide (DMSO), or benzene-was added to the dried ChNS in an Eppendorf tube, followed by homogenization with a Teflon homogenizer.

No species absorbing above $200 \mathrm{~nm}$ was detected in the upper phase, consisting mainly of PBS-methanol, and therefore the upper phase was excluded from further experiments. For some experiments
ChS or ChNS obtained from lipofuscin from 41- to 60- and 61- to 80-year-olds were combined.

\section{Absorption Spectra}

Absorption spectra were measured in a 1- or 0.1-cm optical pathlength quartz cell using a diode-array spectrophotometer (HP 8453; Hewlett Packard, Palo Alto, CA).

\section{Preparation of Liposomes}

Multilamellar liposomes for measurements of photo-induced oxygen uptake consisted of $2 \mathrm{mM}$ dimyristoyl phosphatidylcholine (DMPC), with or without polyunsaturated fatty acids (PUFA) mixture consisting of $1 \mathrm{mM}$ docosatetraenoic methyl ester, $2 \mathrm{mM}$ linoleic acid methyl ester, and $2 \mathrm{mM}$ cholesterol. Liposomes were prepared under anaerobic conditions. A chloroform solution of lipids and/or lipofuscin extracts was dried under argon and then in a vacuum, followed by hydration in PBS. ${ }^{24}$

\section{Monochromatic and Broad-Band Light Irradiation}

Suspensions of pigment granules or extracted material were irradiated either with broad-band visible light derived from a compact-arc, highpressure xenon lamp with a combination of cutoff $(<390 \mathrm{~nm})$ and copper sulfate filters (effective spectral range: 390-620 nm, fluence rate $\sim 23 \mathrm{~mW} / \mathrm{cm}^{2}$ ) or with a narrow-band light, using a combination of broad band, cutoff, and interference filters. The following narrowband irradiations were used: $338 \mathrm{~nm}(16 \mathrm{~nm}) ; 380 \mathrm{~nm}(14 \mathrm{~nm}) ; 404 \mathrm{~nm}$ $(12 \mathrm{~nm}) ; 434 \mathrm{~nm}(10 \mathrm{~nm}) ; 480 \mathrm{~nm}(8 \mathrm{~nm}) ; 494 \mathrm{~nm}(9 \mathrm{~nm}) ; 500 \mathrm{~nm}(8$ $\mathrm{nm}) ; 518 \mathrm{~nm}(6 \mathrm{~nm}) ; 542 \mathrm{~nm}(7 \mathrm{~nm})$, where the value in parentheses corresponds to the full width at half maximum (FWHM). Alternatively, a compact-arc, high-pressure xenon lamp $(75 \mathrm{~W}$; Photon Technologies International Inc., Santa Clara, CA) coupled with a monochromator (Model 101; Photon Technologies International Inc.) was used with a 20-nm bandwidth. Effective fluence rates of narrow-band radiation at the sample surface were within a range of 0.5 to $12 \mathrm{~mW} / \mathrm{cm}^{2}$. Fluence rates of broad-band irradiation were routinely measured with a radiometer (model 65A; Yellow Springs Instruments Co., Yellow Springs, $\mathrm{OH}$ ), and rates of narrow-band irradiation were measured with a calibrated silicon photodiode (Photonics, KK; Hamamatsu, Hamamatsu City, Japan) inside the resonant cavity of an electron spin resonance (ESR) spectrometer.

\section{Photo-Dependent Oxygen Uptake}

Kinetics of oxygen concentration changes in irradiated samples were measured by ESR oximetry. ${ }^{4,25}$ A sample containing $0.1 \mathrm{mM}$ mHCTPO as the nitroxide spin probe was placed in a flat quartz cell $(0.25 \mathrm{~mm})$ in a resonant cavity, and ESR spectra of mHCTPO were collected during their illumination in situ, at ambient temperature. Rates of oxygen uptake were obtained by determining the slope of initial oxygen uptake, where the oxygen concentration decreased linearly with time.

\section{Action Spectra of Photo-Induced Oxygen Uptake}

To obtain action spectra of photo-induced oxygen uptake, the rates of oxygen uptake were normalized to an equal number of incident photons; that is, the rate, expressed in millimolar per second, was divided by the irradiation wavelength (in nanometers) and by the fluence rate (in milliwatts per square centimeter). The values obtained were multiplied by $10^{9}$.

\section{ESR Spectroscopy and Spin Trapping}

ESR spectra were recorded with a spectrometer (ESP 300E; Bruker, Billerica, MA) operating at $9.5 \mathrm{GHz}$ with $100-\mathrm{kHz}$ field modulation. For the detection of superoxide radical anion and other short-lived radicals, lipofuscin granules and ChNS from different age groups were suspended in DMSO, and ChS was solubilized in a DMSO/benzene (1:1) mixture. 5,5-Dimethyl-1-pyrroline- $N$-oxide (DMPO) was used as a spin 


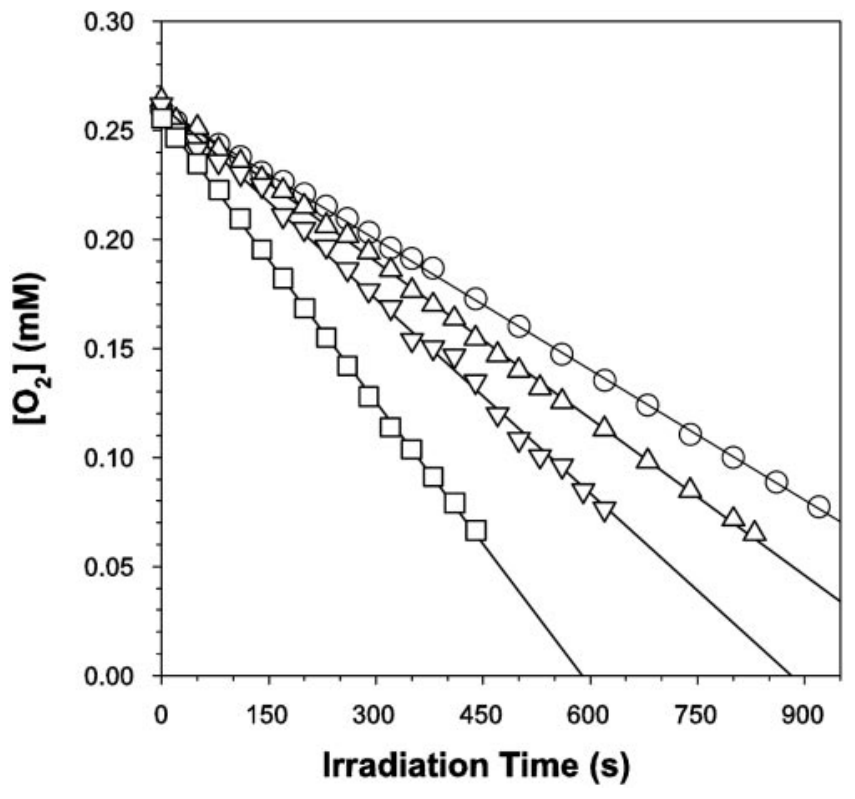

Figure 1. Blue-light-induced oxygen uptake in suspensions of lipofuscin isolated from donors in the following age-groups: 40 years and younger $(\bigcirc), 41$ to $60(\triangle), 61$ to $80(\nabla)$, and 81 to $98(\square)$ years. The concentrations of lipofuscin granules in PBS were adjusted to $7.6 \times 10^{9}$ granules/mL before experiments.

trap, which was added to the sample immediately before a measurement at $0.2 \mathrm{M}$ concentration. ${ }^{26}$ The time course of DMPO adduct formation, and their decay was observed in the dark and during irradiation, with blue light used to distinguish between primary and secondary products.

\section{Singlet Oxygen Detection}

Cholesterol was used as an acceptor of singlet oxygen that gives characteristic oxidation products with singlet oxygen $\mathrm{O}_{2}\left({ }^{1} \Delta_{\mathrm{g}}\right),{ }^{27}$ namely: $3 \beta$-hydroxy- $\alpha \alpha$-cholest-6-ene-5-hydroperoxide $(5 \alpha-\mathrm{OOH}), 3 \beta$ hydroxycholest- 4 -ene- 6 - $\alpha$-hydroperoxide $(6 \alpha-\mathrm{OOH})$, and $3 \beta$-hydroxycholest-4-ene-6- $\beta$-hydroperoxide $(6 \beta$-OOH). Interaction of cholesterol with free radicals leads to generation of $3 \beta$-hydroxycholest-5-ene-7- $\alpha$ hydroperoxide $(7 \alpha-\mathrm{OOH})$ and $3 \beta$-hydroxycholest-5-ene-7- $\beta$-hydroperoxide $(7 \beta-\mathrm{OOH})$. ChNS, at a concentration of $2.4 \mathrm{mg} / \mathrm{mL}$ was suspended in $2 \mathrm{mM}$ cholesterol solution in benzene and irradiated with visible light in a thermostat-controlled chamber $\left(10^{\circ} \mathrm{C}\right)$. At selected irradiation times, samples were collected and centrifuged to sediment the ChNS. Supernatants were dried under argon and solubilized in isopropanol for HPLC-electrochemical detection analysis, as described. $^{28}$

ChS solubilized in benzene at a concentration of $2.0 \mathrm{mg} / \mathrm{mL}$ in the presence of $2 \mathrm{mM}$ cholesterol was used as a positive control for singlet oxygen photogeneration. ${ }^{5,7}$

\section{Statistical Analysis}

Statistical analysis was performed by Student's $t$-test, and linear regression was performed by the method of least squares.

\section{RESUlTS}

\section{Lipofuscin Granules: Photoreactivity in Different Age Groups}

In an earlier study, we showed that, when lipofuscin is irradiated with blue light, lipofuscin components such as lipids are oxidized. The oxidation is accompanied by oxygen uptake, ${ }^{4,11}$ which can thus serve as an oxidation indicator. To establish whether the aerobic photoreactivity of lipofuscin granules undergoes age-related changes, we pooled lipofuscin isolated from RPE cells into four different age groups, adjusted to equal concentrations of granules, and monitored the oxygen uptake during irradiation with blue light $(404 \mathrm{~nm})$. Comparing the rates of oxygen consumption observed for the youngest and the oldest lipofuscin, we found that the process was approximately 1.6 to 2.2 times faster for lipofuscin from donors older than 80 than for donors younger than 40 years old (Fig. 1). Thus, the rate of photo-induced oxygen uptake per granule increased with the age of the lipofuscin donors.

To establish whether age-related changes in lipofuscin photoreactivity depend on wavelength, we measured the action spectra for each of three lipofuscin age groups (Fig. 2; the two middle age groups were pooled for this experiment). For each wavelength studied within the spectral region of 338 to 542 $\mathrm{nm}$, the ratios of oxygen uptake rates were similar: When the youngest and the oldest age group were compared, the ratio varied with wavelength between 1.6 and 2.2, but there were no statistically significant differences between wavelengths.

It has already been established that the aerobic photoexcitation of lipofuscin granules and the ChS extract produces the superoxide radical anion. ${ }^{4,6,11}$ To determine whether photogeneration of superoxide by lipofuscin depends on age, we used ESR spin trapping. When lipofuscin granules were irradiated with visible light in the presence of DMPO in DMSO, the initial ESR spectra consisted mainly of a set of 12 lines with hyperfine coupling constants that could be assigned to the DMPO-OOH adduct (Fig. 3A). ${ }^{26}$ Their initial rate of accumulation depended on concentration and increased monotonically with donor age in the four age groups studied (Fig. 3B). As the rate of adduct decay also increased with donor age, it could be estimated that DMPO-OOH production was greater by a factor of more than 2.1 in the older than 80 group compared with the group younger than 40 years of age. Thus, on a per granule

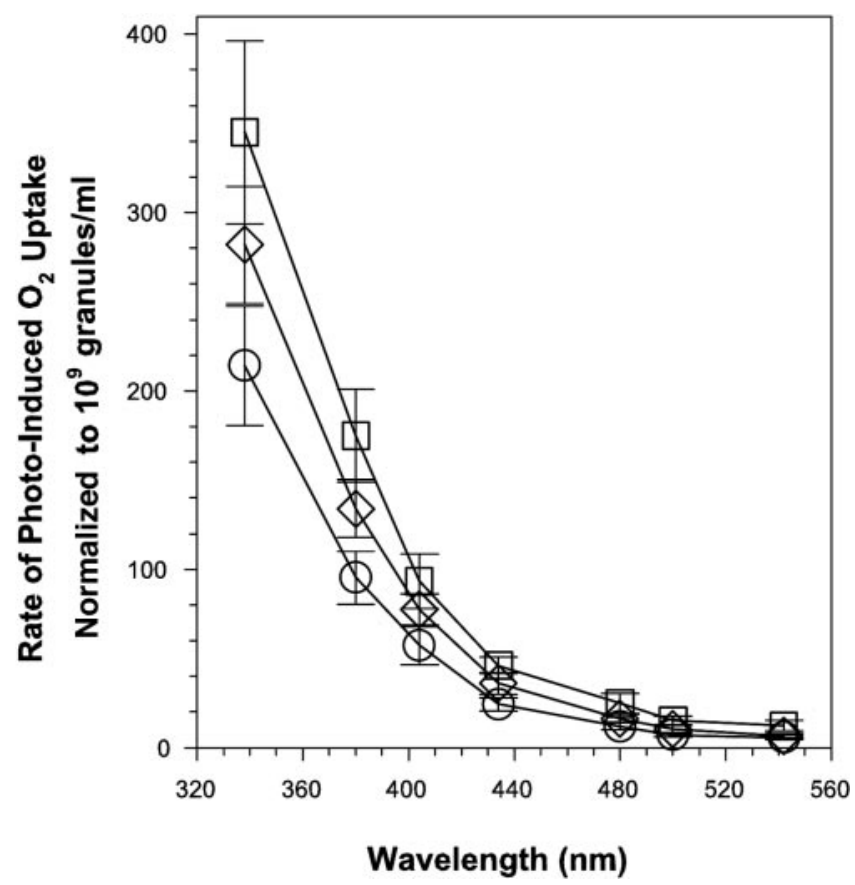

FIGURE 2. Wavelength-dependence of initial rates of photo-induced oxygen uptake in suspensions of lipofuscin granules isolated from donors in the following age-groups: 40 years and younger $(\bigcirc), 41$ to 80 years $(\diamond)$, and older than 80 years $(\square)$. Rates of photo-induced oxygen uptake were normalized to equal number of granules and equal fluxes of incident photons. The granule concentration was as in Figure 1. 

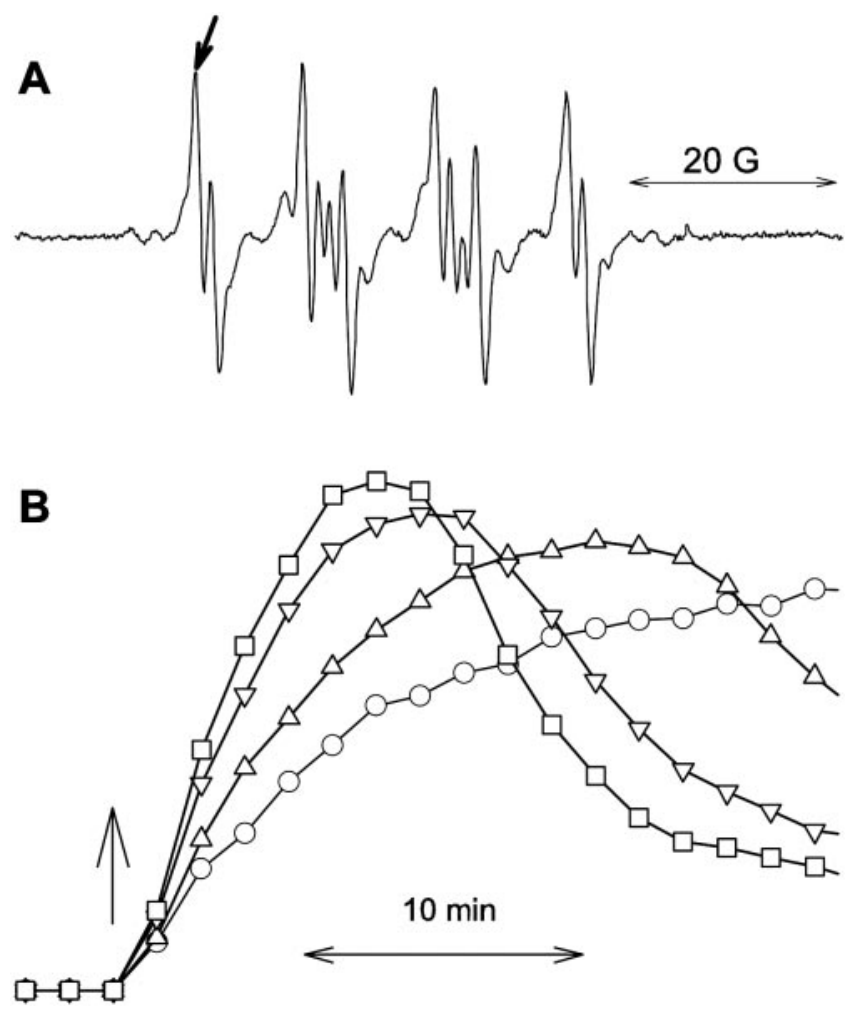

FIGURE 3. ESR spectra for suspensions of lipofuscin granules in the presence of 0.2 M DMPO in DMSO after 12 minutes of irradiation with white light (A). Arrow: component of the spectrum in which amplitude was monitored. Kinetics of changes in amplitudes of spin adducts in suspensions of lipofuscin from different age groups: 40 years and younger $(\bigcirc), 41$ to $60(\triangle), 61$ to $80(\nabla)$, and 81 to $98(\square)$ years. (B). Arrow: beginning of the irradiation. The concentration of granules was $0.9 \times 10^{9}$ granules $/ \mathrm{mL}$. Instrument settings: time constant $0.328 \mathrm{sec}-$ onds, sweep time 160 seconds, microwave power $10 \mathrm{~mW}$, modulation amplitude $1.0 \mathrm{G}$.

basis, both oxygen uptake and superoxide production increased with age.

\section{ChS and ChNS: Content and Photoreactivity in Different Age Groups}

Lipofuscin extraction through Folch's procedure yields ChS lipophilic material and ChNS interphase material. To establish whether the contents of these fractions within lipofuscin granules change with age of lipofuscin donors, their dry masses were weighed. Of note, the content of ChNS per lipofuscin granule significantly increased with age of eye donors by a factor of 1.7 between the youngest and the oldest age group (Fig. 4). The amount of the extracted ChS, in contrast, did not differ significantly between different age groups.

To determine whether the relative quantities of different chromophores within ChS changed with the age of lipofuscin donors, we measured the absorption spectra of ChS from the different age groups. At the same concentration of dry mass per unit of volume, the spectra obtained for ChS from different age groups were very similar to each other over the whole UVA and visible spectral region (Fig. 5A). The action spectra of photo-induced oxygen uptake for ChS from different age groups were also almost identical when normalized to the same dry mass, with the rates increasing steeply with decreasing wavelengths (Fig. 5B). These results indicate a constancy with age in ChS of not only the content of major absorbing chromophores, but also the content of photosensitizer(s).
Moreover, as the absorption spectra of $\mathrm{ChS}$ resemble the action spectra of photo-induced oxygen uptake, it is likely that the absorption spectra of the photosensitizer(s) present in ChS exhibit absorption characteristics similar to those of ChS as a whole. The rates of accumulation and decay of DMPO-OOH adducts photosensitized by ChS from different age groups were not dependent on the age of lipofuscin donors (data not shown).

Although we could not compare the absorption spectra of ChNS from different age groups due to its insolubility, we were able to measure oxygen uptake of ChNS in suspension. Like ChS, ChNS also mediated substantial photo-induced oxygen uptake, with rates that increased with decreasing wavelengths. Again, the action spectra of the rates of photo-induced oxygen uptake for ChNS from different age groups were almost identical when normalized to the same dry mass (Fig. 5C). At longer wavelengths, above $390 \mathrm{~nm}$, the rates of photo-induced oxygen uptake mediated by ChNS equaled or even exceeded the rates for ChS. However, the photoinduced oxygen uptake was markedly smaller at shorter wavelengths than was the case for ChS (Figs. 5B, 5C).

\section{ChNS: Photogeneration of Superoxide Radical Anion and Singlet Oxygen}

To establish whether ChNS photogenerates reactive oxygen species similar to those generated by ChS, we used ESR spin trapping to detect free radicals. When ChNS was irradiated with visible light in the presence of $0.1 \mathrm{M}$ DMPO, the ESR spectra consisted primarily of DMPO-OOH spin adducts (Fig. 6). In samples containing ChNS, the accumulation and decay rates of DMPO-OOH adducts did not differ significantly among different age groups when normalized to equal dry mass of ChNS (data not shown).

We used cholesterol to probe for singlet oxygen production in a sample composed of ChNS pooled from all four age groups. Irradiation of suspensions of ChNS in benzene in the presence of cholesterol resulted in a time-dependent accumulation of cholesterol hydroperoxides (Fig. 7), including characteristic products of interaction of cholesterol with singlet ox-

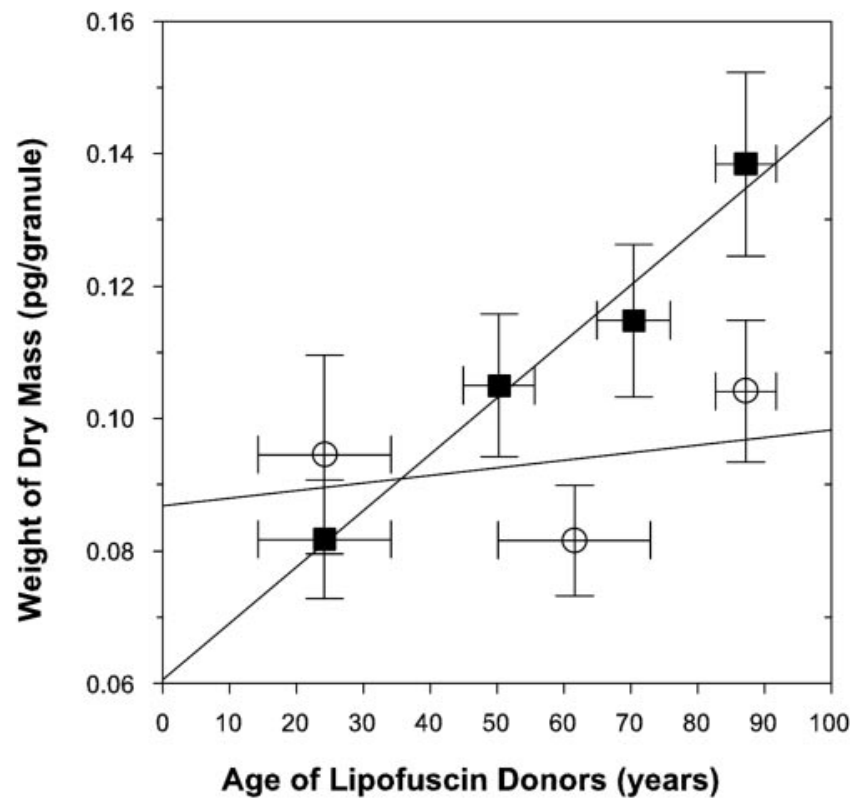

Figure 4. The content of dry mass of ChS (O) and ChNS (ם) extracted from lipofuscin granules from different age groups. Horizontal bars: SD of the donors of the pooled samples; vertical bars: SD of the dry mass measurements. 

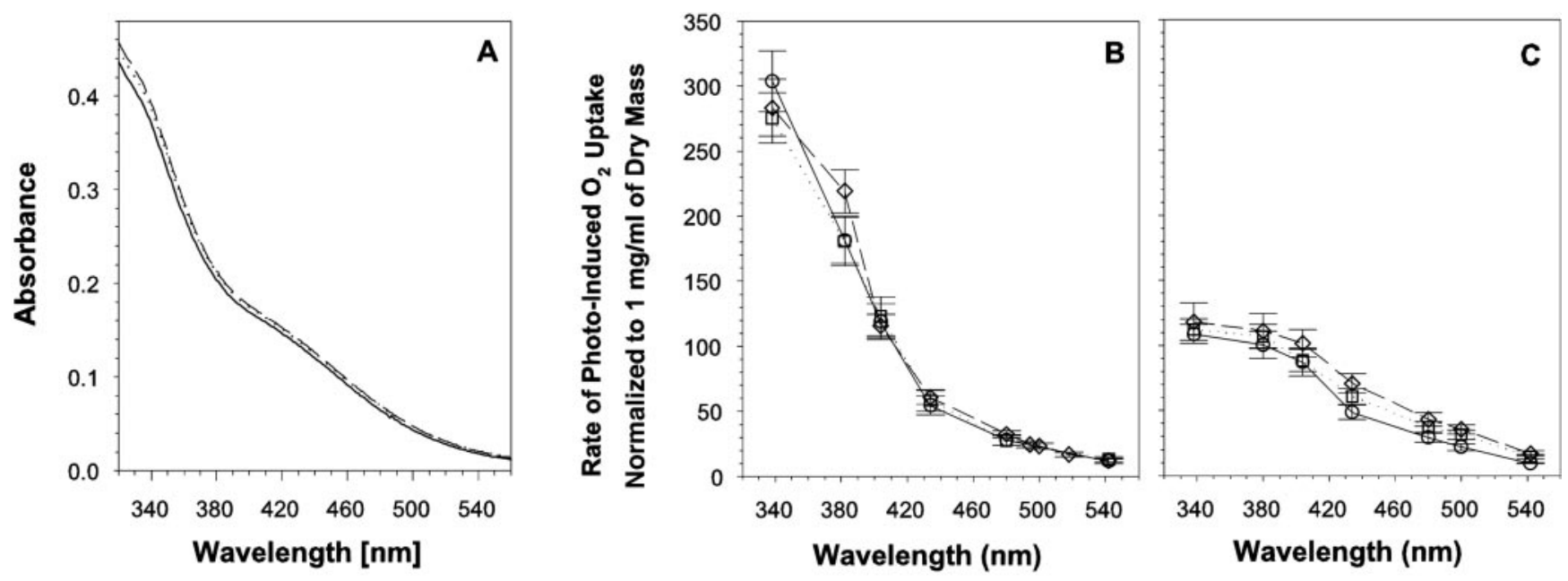

FIGURE 5. Absorption spectra of ChS in benzene $(0.24 \mathrm{mg} / \mathrm{mL}$ dry mass) in a $0.1-\mathrm{cm}$ cell (A) and wavelength dependences of initial rates of photo-induced oxygen uptake of ChS in DMPC liposomes (B) or ChNS suspensions (C) in PBS isolated from donors in the following age groups: younger than 40 years $(\bigcirc$, solid line $), 41$ to $80(\triangle$, dashed line), and 81 to $98(\nabla$, dotted line) years. Rates of photo-induced oxygen uptake were normalized to equal dry mass content and equal fluxes of incident photons.

ygen: $5 \alpha-\mathrm{OOH}, 6 \alpha-\mathrm{OOH}$, and $6 \beta-\mathrm{OOH}$. No accumulation of $5 \alpha-\mathrm{OOH}, 6 \alpha-\mathrm{OOH}$, or $6 \beta-\mathrm{OOH}$ was observed when the sample was incubated in the dark (data not shown), or if the sample was irradiated in the absence of ChNS (Fig 7d). Thus, ChNS clearly photogenerates singlet oxygen.

The direct meaningful comparison on the yields of superoxide and singlet oxygen photogeneration is not possible, due to the different states of ChNS (suspension of particles) and ChS (solution). First of all, the absorption cross sections are not comparable. Second, DMPO or cholesterol competes with lipofuscin components for reactive oxygen species under completely different conditions. In the case of ChS solution, the reactive oxygen species are photogenerated directly in solution, whereas for a ChNS suspension, it may be expected that reactive oxygen species need to diffuse out of the particle to be trapped by DMPO or cholesterol in solution.

\section{Role of ChS and ChNS in Lipofuscin Photoreactivity}

Both ChS and ChNS induced substantial photo-dependent oxygen uptake; however, when their rates were normalized to the lipofuscin granule content from different age groups and

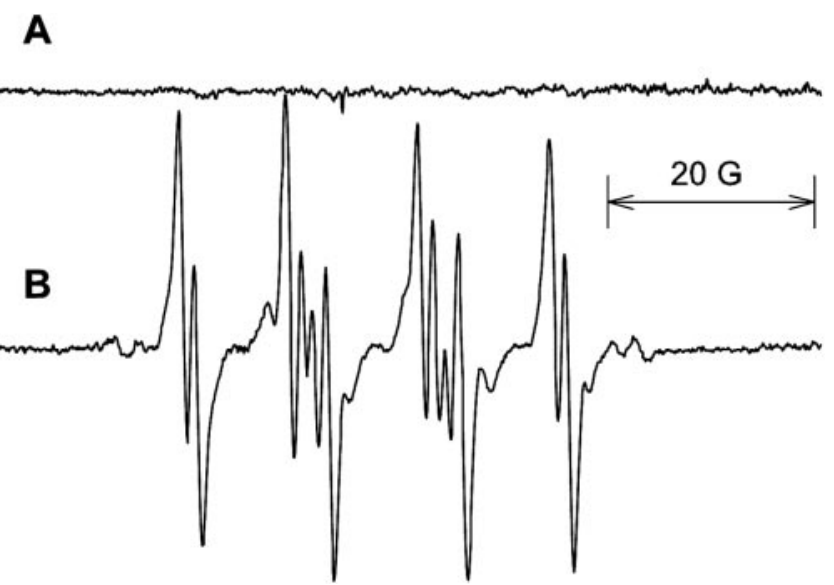

FIGURE 6. ESR spectra for ChNS $(0.3 \mathrm{mg} / \mathrm{mL})$ in the presence of $0.2 \mathrm{M}$ DMPO in DMSO before (A) and after 12 minutes of irradiation with white light (B). Other experimental conditions are as in Figure 3. added arithmetically, the rates were 3 to 7 times lower than the corresponding values in action spectra measured for lipofuscin granules (Fig. 8). A possible reason for the decreased rates may be dilution or complete separation of substrates of oxidation from photosensitizers. To test this possibility, the ChS and ChNS fractions were recombined in DMPC liposomes, and the rates of photo-induced oxygen uptake were compared with the rates for ChS and ChNS separately and their arithmetical addition (Fig. 9). Although when excited with wavelengths

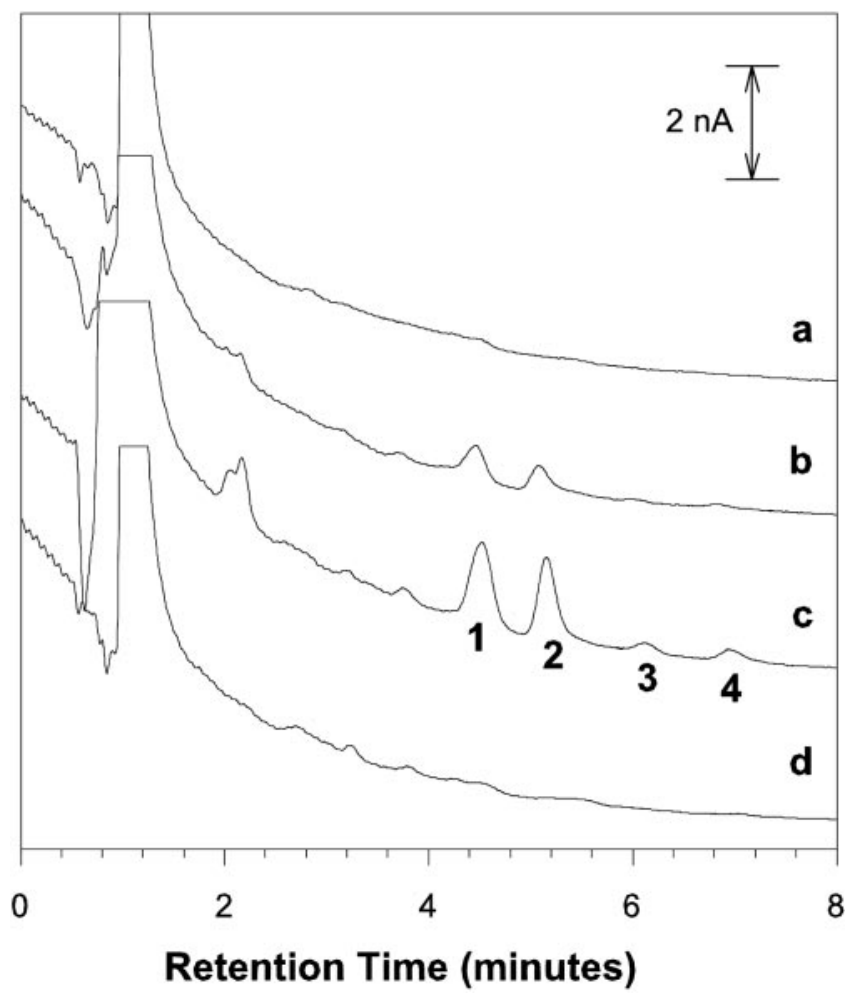

Figure 7. HPLC-EC detection of cholesterol hydroperoxides in samples irradiated with white light in the presence $(a, b, c)$ or in the absence of ChNS (d) for 0 (a), 15 (b) or 30 minutes (c, d). The indicated signals correspond to: (1) $7 \alpha / \beta-\mathrm{OOH}$, (2) $5 \alpha-\mathrm{OOH}$, (3) $6 \alpha-\mathrm{OOH}$, (4) $6 \beta-\mathrm{OOH}$. 

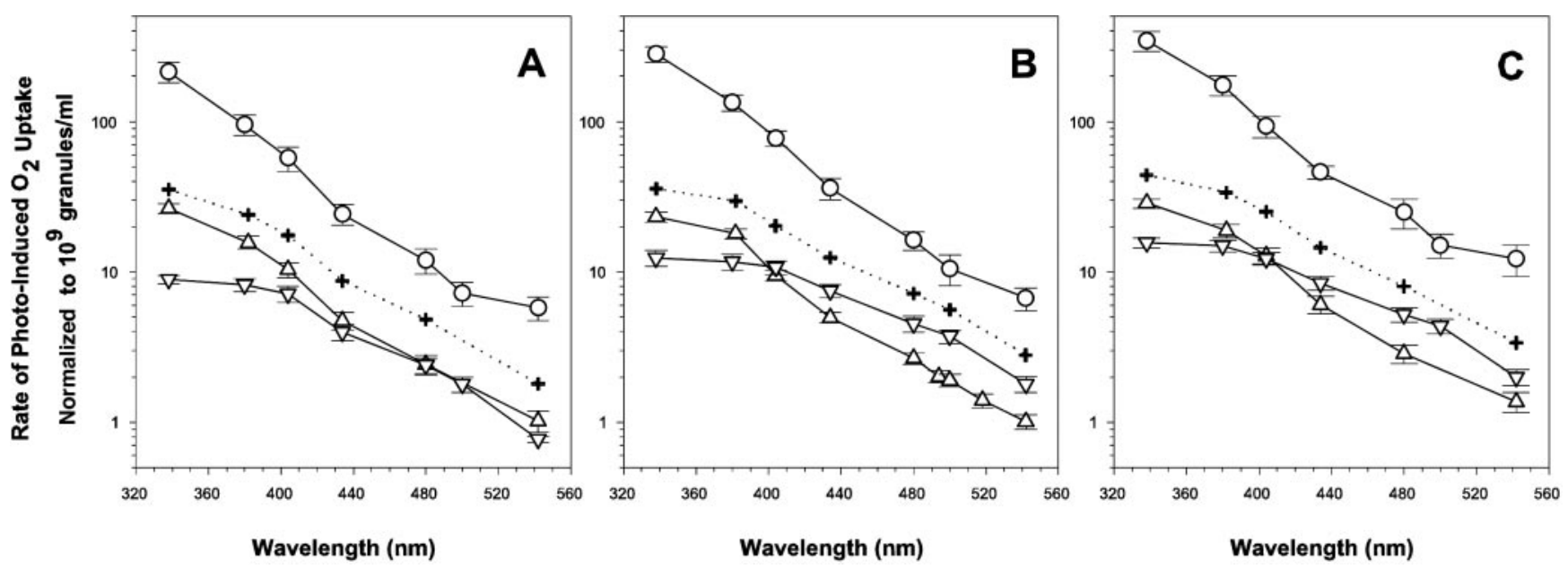

FIGURE 8. Wavelength-dependence of initial rates of photo-induced oxygen uptake in suspensions of lipofuscin granules $(\bigcirc)$, ChS $(\triangle)$, ChNS $(\nabla)$, and the arithmetical addition of the rates $(+)$ for donors aged younger than 40 years $(\mathbf{A}), 41$ to 80 years $(\mathbf{B})$, and older than 80 years $(\mathbf{C})$. $(\mathbf{B})$ Based on the results presented in Figures 2, 4, and 5. Rates of photo-induced oxygen uptake were normalized to equal fluxes of incident photons and to $10^{9}$ granules/mL or corresponding content of ChS and ChNS in suspensions of lipofuscin granules.

above $380 \mathrm{~nm}$, the recombined material had photo-induced oxygen uptake rates almost identical with those obtained by the arithmetical addition of the values for ChS and ChNS, excitation by $340 \mathrm{~nm}$ light resulted in rates up approximately 1.6 times higher (Fig. 9).

To determine whether added lipids or proteins may become substrates for photooxidation by ChS and ChNS, we compared the rates of photo-induced oxygen uptake in the presence and absence of added polyunsaturated lipids and proteins. In the presence of additional oxidation substrates, UVA $(340 \mathrm{~nm})$ and blue-light $(410 \mathrm{~nm})$ induced increased oxygen uptake for both fractions (Fig. 10). Irradiation with $410 \mathrm{~nm}$ light increased the oxygen uptake rate by a factor of 1.7 in the presence of

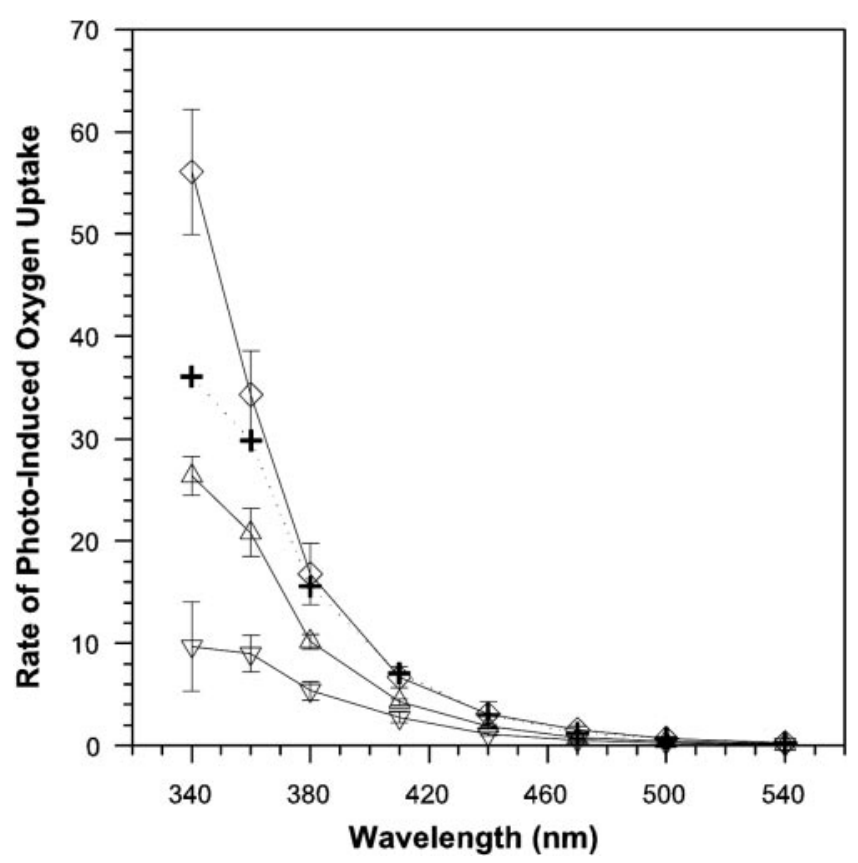

Figure 9. Comparison of the action spectra of initial rates of photoinduced oxygen uptake in suspensions of DMPC liposomes containing both ChS and ChNS (reconstituted lipofuscin, $\diamond$ ), and the arithmetic addition $(+)$ of the rates measured separately for ChS $(\triangle)$ and ChNS $(\nabla)$ in DMPC liposomes. additional oxidation substrates for both $\mathrm{ChS}$ and ChNS. Of note, during irradiation with $340 \mathrm{~nm}$, addition of polyunsaturated lipids and bovine serum albumin (BSA) to ChS induced only a 1.4-fold increase in the oxygen uptake rate, whereas for ChNS, the increase was nearly 3-fold.

There is a possibility that photosensitizer(s) are localized in liposomes in proximity of the ChS components available for oxidation. Therefore, it might be speculated that further enrichment of liposomes in PUFA and/or BSA does not substantially increase the local concentration of the oxidation substrate in proximity of photosensitizer(s), so they exhibit a small effect on the initial rates of the photo-induced oxygen uptake as seen in Figure 10B.

\section{Discussion}

\section{Age-Related Increase in Lipofuscin Photoreactivity}

The results obtained in this study clearly demonstrate that the photoreactivity of lipofuscin granules increases with donor age, as shown by the increased rates for photo-induced oxygen uptake and accumulation of DMPO-OOH spin adducts. At least three age-related factors may contribute to these increased rates: (1) an increased content of photosensitizers that are involved in the generation of oxygen reactive species, (2) a higher content of substrates of photosensitized oxidation, and/or (3) a decreased content of endogenous antioxidants present in the lipofuscin granule. Indeed, the presence of all three lipofuscin constituents has been demonstrated in the ChS fraction of lipofuscin: unidentified photosensitizers, ${ }^{5-7}$ PUFAs, ${ }^{20}$ and vitamin E (Sarna T, et al. IOVS 2001; 42 :ARVO Abstract 4095).

The observed age-related increase in photoreactivity of lipofuscin granules reflects age-related changes within the whole population of lipofuscin granules. It should be stressed that the population of lipofuscin at any age may be very heterogenous as indicated by substantial differences in emission properties among lipofuscin granules. ${ }^{29,30}$ Heterogeneity is also present within single granules, as was shown by comparative measurements of topography and fluorescence by near-field scanning optical microscopy. ${ }^{29,30}$

The composition of lipofuscin was also found to change with age; specifically, the amount of ChNS per granule in- 

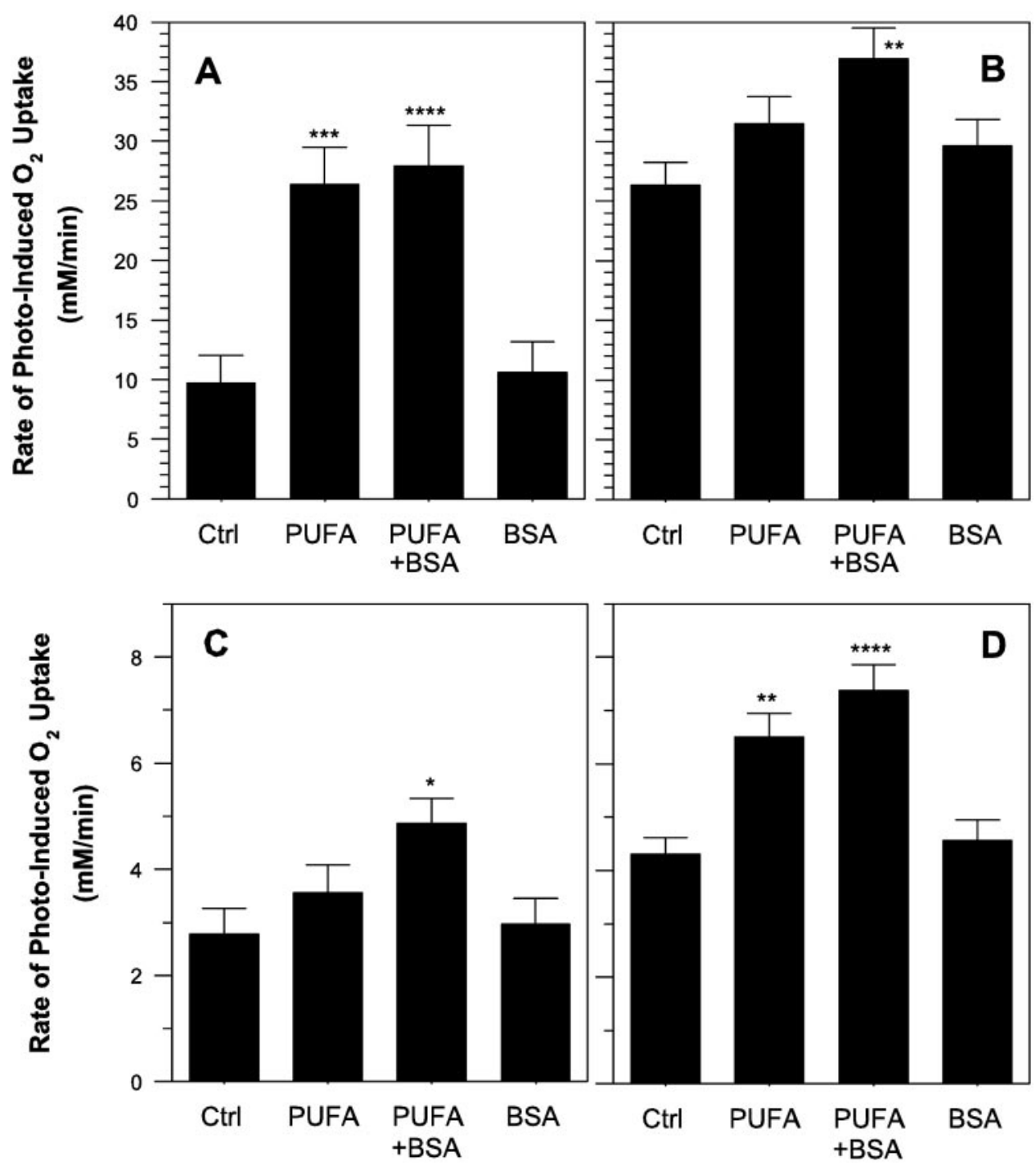

FIGURE 10. Rates of UVA- $(340 \mathrm{~nm}$; A, B) or blue (410 nm; C, D)-lightinduced oxygen uptake in suspensions of ChNS $(\mathbf{A}, \mathbf{C})$ or $\mathrm{ChS}(\mathbf{B}, \mathbf{D})$ in DMPC liposomes (Ctrl) or with additional substrate of oxidation PUFA, both PUFA and BSA, or BSA. The rates of photo-induced oxygen uptake by PUFA and BSA alone were negligible under the conditions used. Data are the mean $\pm \mathrm{SD}$ of results in at least three experiments. Statistically significant differences from the control are indicated ${ }^{*} P=0.02$, ${ }^{* *} P=0.05,{ }^{* * *} P=0.002$, and ${ }^{* * * * *} P=$ 0.001 .

creased, while the amount of ChS remained the same. ChS has already been shown to contain photosensitizers, to exhibit photo-induced oxygen uptake, and to photogenerate superoxide and singlet oxygen. Now, we have demonstrated that ChNS also exhibits photo-induced oxygen uptake and photogeneration of DMPO-OOH spin adducts and singlet oxygen, indicating that it also contains photosensitizers. We cannot exclude the possibility that the chromophores responsible for lipofuscin photoreactivity are similar in both ChS and ChNS, but are bound to different groups of lipids and/or proteins, which to some extent may modify their physicochemical properties.

The photoinduced rates of oxygen uptake mediated by ChNS are comparable to those of ChS, particularly within the blue-green light spectral region, when normalized to the same dry mass. When normalized to equal dry mass, neither exhibits significant age-related changes in photoreactivity. This observation may indicate that the content of photosensitizers, substrates of oxidation, and antioxidants remains almost the same within each fraction of lipofuscin, and that the observed changes in lipofuscin photoreactivity are due to age-related changes in the relative amounts of ChS and ChNS. Because in our study we observed a significant increase only in the content of ChNS in lipofuscin granules with age, whereas the content of ChS did not exhibit any significant changes with age, we postulate that the apparent increased photoreactivity of lipofuscin granules is related to the increased content of ChNS.

One of the manifestations of increased photoreactivity with age is the increased accumulation of DMPO-OOH spin adducts. As DMPO-OOH increases with age by a factor of 2.1, and the competitive scavenging of superoxide radical anion by lipofuscin granules is negligible, ${ }^{31}$ it may be concluded that the rate of photogeneration of superoxide radical anion by photoexcited lipofuscin also increases with aging. Although both photoexcited ChS and ChNS can generate superoxide, it is difficult to compare their efficiencies due to the different solvents used for solubilization of ChS and ChNS, which yields a different degree of solubilization of the two lipofuscin fractions and different stabilities of the free radicals. When normalized to equal dry mass, the efficiency of photogeneration of superoxide radical anion by ChS or ChNS did not depend on the age of lipofuscin donors, therefore the age-dependent increase in the efficiency of photogeneration of superoxide radical anion by lipofuscin granules must be due to the increased content of ChNS in the lipofuscin granule.

The increased ability of lipofuscin from aged RPE to photogenerate superoxide may have serious consequences in the aged RPE. Dismutation of superoxide radical anion, greatly enhanced by superoxide dismutase or low $\mathrm{pH}$ (typical for lysosomes), leads to generation of hydrogen peroxide. How- 
ever, catalase activity in the human RPE decreases with aging. ${ }^{32}$ Moreover, catalase is inhibited during irradiation in vitro with blue light, and this effect is exacerbated in the presence of lipofuscin. ${ }^{8}$ Therefore, as the efficacy of the antioxidant system protecting against hydrogen peroxide may be diminished, it is more likely that photogenerated hydrogen peroxide may exert deleterious effects in aged RPE cells.

\section{The Role of ChS and ChNS in the Photoreactivity of Lipofuscin}

Both ChS and ChNS exhibit substantial photoreactivity: On photoexcitation under aerobic conditions they generate superoxide and singlet oxygen and lead to oxygen consumption. However, when compared with intact lipofuscin granules, the sums of their rates of photo-induced oxygen uptake are three to seven times lower than the rates observed in suspensions of lipofuscin granules.

This discrepancy may be largely explained by the decreased availability of oxidation substrates in the suspensions of $\mathrm{ChS}$ in liposomes and of ChNS. For suspensions of lipofuscin granules, no more than $3 \%$ of the oxygen consumed during irradiation with blue light accumulates as hydrogen peroxide, ${ }^{11}$ a typical product of dismutation of superoxide anion radicals. Most of the oxygen consumed is used in oxidation of lipofuscin components. ${ }^{11}$ Both ChS and ChNS can induce photo-oxidation of added lipids and proteins. However, solubilization of ChS in solution or DMPC liposomes results in dilution of the substrates of oxidation, whereas suspensions of ChNS are altogether devoid of oxidation substrates such as lipids. Therefore, it may be the reduced concentration of substrate for oxidation in the proximity of photosensitizers that is responsible for the fact that the sums of the rates of photo-induced oxygen uptake observed for ChS and ChNS are several times lower than that observed for the corresponding number of lipofuscin granules.

It is important to have oxidizable substrate present because of the short lifetime of one of the reactive oxygen species: singlet oxygen. Singlet oxygen is one of the reactive oxygen species responsible for the oxidation of extragranular lipids mediated by photoexcited lipofuscin granules. ${ }^{4}$ It is a powerful oxidant of proteins and unsaturated lipids, ${ }^{33}$ provided the substrates of oxidation are in the proximity of the photosensitizers. Otherwise, singlet oxygen will thermally deactivate to its ground state, as its lifetime in aqueous solutions is only approximately $4 \mu \mathrm{s} .{ }^{34}$ Therefore, even though both ChS and ChNS photosensitize singlet oxygen formation, oxygen uptake would follow only if an appropriate substrate of oxidation is present in proximity.

The paucity of oxidizable substrates can also explain much of the single biggest difference in photoreactivity between the ChS fraction and ChNS: their action spectra in the UV. The action spectrum for ChS follows a shape similar to that of lipofuscin granules, although quantitatively the yields are smaller. The action spectrum of ChNS, however, although similar to lipofuscin granules at longer wavelengths, is markedly lower at wavelengths shorter than $410 \mathrm{~nm}$, unless additional oxidation substrates are added. In the latter case, the action spectrum of ChNS approaches the shape of the lipofuscin granule spectrum for wavelengths as short as $340 \mathrm{~nm}$.

When ChNS is irradiated with $340-\mathrm{nm}$ light, the rate of photo-induced oxygen uptake increases by a factor of 3 in the presence of polyunsaturated lipids, which appear to be a particularly good oxidation substrate for ChNS photoexcited with UV light. However, when the excitation wavelength is $410 \mathrm{~nm}$, the increase in the presence of additional lipids and proteins is only 1.7 -fold. When the two fractions of lipofuscin are combined, the oxygen uptake rate increases synergistically when excited at $340 \mathrm{~nm}$ and only additively when excited with wavelengths of $410 \mathrm{~nm}$ and higher. This wavelength-dependence of the photoreactivity of ChNS may be related to a quantum yield of singlet oxygen generation that is different for UV excitation than for blue-green light. This is the case for ChS, where the quantum yield of singlet oxygen decreased by a factor of 1.7 when photoexcited with blue light (420, 430, and $440 \mathrm{~nm}$ ) as opposed to $355 \mathrm{~nm}^{7}$

Although the number of lipofuscin granules and their shortwavelength light-induced photoreactivity increase with age, the transmission properties of the anterior segment of the eye, mainly of the lens, also undergo age-related changes. ${ }^{1}$ Although UVA light is completely blocked by the lens in a 20 -year-old adult, with aging, more and more short-wavelength visible light is gradually absorbed by the lens, and therefore less blue light reaches the retina. Thus, it may be speculated that despite the increased photoreactivity of lipofuscin with increasing donor age, the risk of photooxidative damage to RPE cells is mitigated by the decreased flux of incident blue light. However, the situation is different in individuals after cataract surgery where a naturally yellowed lens is replaced with a transparent artificial lens. Thus, their retinas become exposed to fluxes of light similar to those in young individuals.

\section{Conclusions}

The age-related increase in the photoreactivity of lipofuscin granules together with an increased number of lipofuscin granules may impose a greater risk of photooxidative damage in the aged RPE compared with the young RPE. Moreover, the chloroform insoluble components of lipofuscin seem to play a substantial role in the photoreactivity of lipofuscin granules, a role whose impact increases with the age of the lipofuscin donors.

\section{Acknowledgments}

The authors thank Ann Motten for critical reading and amendments to the manuscript.

\section{References}

1. Boulton M, Rozanowska M, Rozanowski B. Retinal photodamage. $J$ Photochem Photobiol B. 2001;64:144-161.

2. Eldred GE. Lipofuscin and other lysosomal storage deposits in the retinal pigment epithelium. In: Marmor MF, Wolfensberger TJ, eds. The Retinal Pigment Epithelium Function and Disease. New York: Oxford University Press; 1998:651-668.

3. Boulton M, Dontsov A, Jarvisevans J, Ostrovsky M, Svistunenko D. Lipofuscin is a photoinducible free-radical generator. J Photochem Photobiol B. 1993;19:201-204.

4. Rozanowska M, Jarvis-Evans J, Korytowski W, Boulton ME, Burke JM, Sarna T. Blue light-induced reactivity of retinal age pigment: in-vitro generation of oxygen-reactive species. J Biol Chem. 1995; 270:18825-18830.

5. Gaillard ER, Atherton SJ, Eldred G, Dillon J. Photophysical studies on human retinal lipofuscin. Photochem Photobiol. 1995;61:448453.

6. Reszka K, Eldred GE, Wang RH, Chignell C, Dillon J. The photochemistry of human retinal lipofuscin as studied by EPR. Photochem Photobiol. 1995;62:1005-1008.

7. Rozanowska M, Wessels J, Boulton M, et al. Blue light-induced singlet oxygen generation by retinal lipofuscin in non-polar media. Free Radic Biol Med. 1998;24:1107-1112.

8. Wassell J, Davies S, Bardsley W, Boulton M. The photoreactivity of the retinal age pigment lipofuscin. J Biol Chem. 1999;274:2382823832.

9. Davies S, Elliott MH, Floor E, et al. Photocytotoxicity of lipofuscin in human retinal pigment epithelial cells. Free Radic Biol Med. 2001;31:256-265. 
10. Pawlak A, Rozanowska M, Zareba M, Lamb LE, Simon JD, Sarna T Action spectra for the photoconsumption of oxygen by human ocular lipofuscin and lipofuscin extracts. Arch Biochem Biophys. 2002; 403:59-62.

11. Rozanowska M, Korytowski W, Rozanowski B, et al. Photoreactivity of aged human RPE melanosomes: a comparison with lipofuscin. Invest Opbthalmol Vis Sci. 2002;43:2088-2096.

12. Parish CA, Hashimoto M, Nakanishi K, Dillon J, Sparrow J. Isolation and one-step preparation of A2E and iso-A2E, fluorophores from human retinal pigment epithelium. Proc Natl Acad Sci USA. 1998; 95:14609-14613.

13. Schutt F, Davies S, Kopitz J, Holz FG, Boulton ME. Photodamage to human RPE cells by A2-E, a retinoid component of lipofuscin. Invest Opbthalmol Vis Sci. 2000;41:2303-2308.

14. Sparrow JR, Nakanishi K, Parish CA. The lipofuscin fluorophore A2E mediates blue light-induced damage to retinal pigmented epithelial cells. Invest Ophthalmol Vis Sci. 2000;41:1981-1989.

15. Suter M, Reme C, Grimm C, et al. Age-related macular degeneration: the lipofuscin component $N$-retinyl- $N$-retinylidene ethanolamine detaches proapoptotic proteins from mitochondria and induces apoptosis in mammalian retinal pigment epithelial cells. J Biol Chem. 2000;275:39625-39630.

16. Sparrow JR, Zhou J, Ben-Shabat S, Vollmer H, Itagaki Y, Nakanishi $\mathrm{K}$. Involvement of oxidative mechanisms in blue-light-induced damage to A2E-laden RPE. Invest Ophthalmol Vis Sci. 2002;43: 1222-1227.

17. Cantrell A, McGarvey DJ, Roberts J, Sarna T, Truscott TG. Photochemical studies of A2-E. J Pbotochem Pbotobiol B. 2001;64:162165 .

18. Lamb LE, Ye T, Haralampus-Grynaviski NM, et al. Primary photophysical properties of A2E in solution. J Phys Chem B. 2001;105: 11507-11512.

19. Pawlak A, Wrona M, Rozanowska M, et al. Comparison of the aerobic photoreactivity of A2E with its precursor retinal. Pbotochem Pbotobiol. 2003;77:253-258.

20. Bazan HEP, Bazan NG, Feeney-Burns L, Berman ER. Lipids in human lipofuscin-enriched subcellular-fractions of 2 age populations: comparison with rod outer segments and neural retina. Invest Opbthalmol Vis Sci. 1990;31:1433-1443.

21. Docchio F, Boulton M, Cubeddu R, Ramponi R, Barker PD. Agerelated changes in the fluorescence of melanin and lipofuscin granules of the retinal-pigment epithelium: a time-resolved fluorescence spectroscopy study. Photochem Pbotobiol. 1991;54:247253.
22. Boulton M, Docchio F, Dayhaw-Barker P, Ramponi R, Cubeddu R. Age-related changes in the morphology, absorption and fluorescence of melanosomes and lipofuscin granules of the retinal pigment epithelium. Vision Res. 1990;30:1291-1303.

23. Folch J, Lees M, Sloane SGH. A simple method for the isolation and purification of total lipids from animal tissues. J Biol Chem. 1957; 226:497-509.

24. Szoka FJ, Papahadjopoulos D. Comparative properties and methods of preparation of lipid vesicles (liposomes). Ann Rev Biopbys Bioeng. 1980;9:467-508.

25. Halpern HJ, Peric M, Nguyen TD, et al. Selective isotopic labeling of a nitroxide spin label to enhance sensitivity for T2 oxymetry. 1990;90:40 - 51.

26. Buettner GR. Spin trapping: electron-spin-resonance parameters of spin adducts. Free Radic Biol Med. 1987;3:259-303.

27. Korytowski W, Girotti AW. Singlet oxygen adducts of cholesterol: photogeneration and reductive turnover in membrane systems. Photochem Pbotobiol. 1999;70:484-489.

28. Korytowski W, Bachowski GJ, Girotti AW. Chromatographic separation and electrochemical determination of cholesterol hydroperoxides generated by photodynamic action. Anal Biochem. 1991;197:149-156.

29. Krogmeier JR, Clancy CMR, Pawlak A, et al. Mapping the distribution of emissive molecules in human ocular lipofuscin granules with near-field scanning optical microscopy. J Microsc. 2001;202: 386-390.

30. Clancy CMR, Krogmeier JR, Pawlak A, et al. Atomic force microscopy and near-field scanning optical microscopy measurements of single human retinal lipofuscin granules. J Phys Chem B. 2000; 104:12098-12101.

31. Dontsov AE, Sakina NL, Ostrovskii MA. Lipofuscin granules and melanosomes from human retinal pigment epithelium have opposite roles in photooxidation of cardiolipin [in Russian]. Biofizika. 1999; $44: 880-886$

32. Liles MR, Newsome DA, Oliver PD. Antioxidant enzymes in the aging human retinal-pigment epithelium. Arch Opbthalmol. 1991; 109:1285-1288.

33. Foote CS. Photooxidation of biological model compounds. In: Rodgers MAJ, Powers EL, eds. Oxygen and Oxy-Radicals in Chemistry and Biology. New York: Academic Press; 1981:425439.

34. Wessels JM, Rodgers MAJ. Detection of the $\mathrm{O}_{2}\left({ }^{1} \Delta_{\mathrm{g}}\right) \rightarrow \mathrm{O}_{2}\left({ }^{3} \Sigma_{\mathrm{g}}{ }^{-}\right)$ transition in aqueous environments: a Fourier-transform near-infrared luminescence study. J Phys Chem. 1995;99:15725-15727. 